\title{
Stochastic Analysis of Natural Convection in Vertical Channels with Random Wall Temperature
}

\author{
Ryoichi Chiba \\ Department of Mechanical Systems Engineering, National Institute of Technology, Asahikawa College, 2-2-1-6 Shunkodai, \\ Asahikawa 071-8142, Japan \\ Correspondence should be addressed to Ryoichi Chiba; chiba@asahikawa-nct.ac.jp
}

Received 31 May 2017; Accepted 17 July 2017; Published 13 August 2017

Academic Editor: Sergey A. Suslov

Copyright (C) 2017 Ryoichi Chiba. This is an open access article distributed under the Creative Commons Attribution License, which permits unrestricted use, distribution, and reproduction in any medium, provided the original work is properly cited.

\begin{abstract}
This study attempts to derive the statistics of temperature and velocity fields of laminar natural convection in a heated vertical channel with random wall temperature. The wall temperature is expressed as a random function with respect to time, or a random process. First, analytical solutions of the transient temperature and flow velocity fields for an arbitrary temporal variation in the channel wall temperature are obtained by the integral transform and convolution theorem. Second, the autocorrelations of the temperature and velocity are formed from the solutions, assuming a stationarity in time. The mean square values of temperature and velocity are computed under the condition that the fluctuation in the channel wall temperature can be considered as white noise or a stationary Markov process. Numerical results demonstrate that a decrease in the Prandtl number or an increase in the correlation time of the random process increases the level of mean square velocity but does not change its spatial distribution tendency, which is a bell-shaped profile with a peak at a certain horizontal distance from the channel wall. The peak position is not substantially affected by the Prandtl number or the correlation time.
\end{abstract}

\section{Introduction}

Recently, as reliability gains increasing importance in the design phase of thermal systems, conventional deterministic heat transfer analysis alone is not sufficient; analysis that considers uncertainties included in the systems themselves and/or thermal environments (in other words, heating/cooling conditions) is required. In general, accurately predicting the thermal or mechanical loads acting on components in high-temperature apparatus is very difficult. This alludes to the fact that many uncertain factors exist in the design of such apparatus [1]. In this situation, the temperature fields in solid objects and/or working fluid should be estimated stochastically.

There has been an interest in analysing and quantifying the effects of uncontrollable random factors on the fluid flow and heat transfer performances of thermal systems. To estimate the effects quantitatively, probabilistic methods have been applied to heat transfer problems. Existing literature about the application of the methods to heat conduction problems is summarised in review articles [2-4], and therefore we do not enter further into the subject here. On the other hand, focusing on the application to convective heat transfer problems, one can find early studies of stochastic forced convection with heat source and initial and boundary conditions being white noises $[5,6]$. Subsequently, other researchers investigated the effects of random temperature and velocity of a moving wall on the temperature field in a Couette flow [7], the effects of boundary temperature and boundary topology modelled by random fields on the temperature and velocity fields of natural convection in a square domain $[8,9]$, and the effects of boundary temperature, which was assumed to be a random field, on the Nusselt numbers of mixed convection in a horizontal channel [10].

However, the effects of randomly fluctuating the temperature of a bounding wall on the fluid flow and thermal performance of natural convection in channels have not been investigated. The temperature fluctuations at the wall are not necessarily zero in real situations [11], which are caused by various natural noises. In addition, the effects of wall temperature fluctuations on the evolution of the fluid flow 


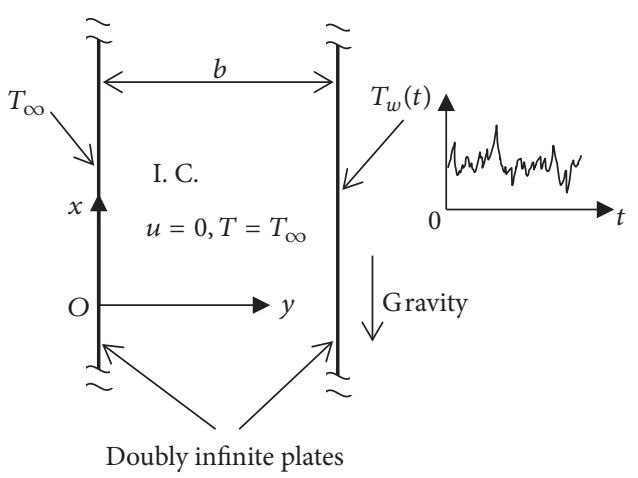

FIGURE 1: Schematic of a vertical parallel-plate channel with random wall temperature.

and heat transfer are significant in internal natural convection [12].

In this paper, we address the stochastic natural convection problem of viscous fluid in an infinite vertical channel with temporally random wall temperature. Under the assumption that the wall temperature is a stationary random process, the autocorrelation functions are analytically derived for the fluid temperature and flow velocity. Numerical calculations are performed under the condition that the wall temperature is expressed either as white noise or as a stationary Markov process. We quantify the effects of the Prandtl number and the correlation time of the wall temperature fluctuation on the mean square values (i.e., second moments) of the fluid temperature and velocity. This stochastic problem may also be solved numerically by applying a variance propagation algorithm based on the finite element formulation [13]. However, our analytical solutions to the second moments are mathematically sounder.

\section{Analysis of Natural Convection Problem}

Let us consider the transient laminar natural convection of a viscous incompressible fluid in an infinite vertical channel, as shown schematically in Figure 1. Two flat plates having infinite width and length (i.e., doubly infinite plates) are placed vertically with a separation distance of $b$ in the fluid. Initially, the fluid is quiescent at uniform temperature $T_{\infty}$, and the vertical plates are maintained at the same temperature $T_{\infty}$. It is assumed that the temperature $T_{w}(t)$ of the plate located at $y=b$ fluctuates randomly for time $t>0$ whereas the other plate is kept constant at $T_{\infty}$. The physical properties of the fluid are constant except for its density; density variation is considered only in the buoyancy term, which is a linear function of temperature (i.e., the usual Boussinesq approximation). Viscous dissipation effects are neglected.

In this physical situation, the fluid motion can be regarded as one-dimensional (1D) with only one nonzero velocity component, $u$, varying with $y$ and $t$. Due to the $1 \mathrm{D}$ nature, the continuity equation is trivially satisfied. The energy equation for the fluid reduces to the $1 \mathrm{D}$ conduction equation. Thus, the momentum equation for the $x$-direction and the energy equation are given, respectively, as follows [14-16]:

$$
\begin{aligned}
\frac{\partial u}{\partial t} & =\nu \frac{\partial^{2} u}{\partial y^{2}}+g \beta\left(T-T_{\infty}\right), \\
\frac{1}{\alpha} \frac{\partial T}{\partial t} & =\frac{\partial^{2} T}{\partial y^{2}} .
\end{aligned}
$$

The imposed initial and boundary conditions are as follows:

$$
\begin{aligned}
& u=0 \quad \text { at } t=0, \\
& T=T_{\infty} \quad \text { at } t=0, \\
& u=0 \quad \text { at } y=0, \\
& T=T_{\infty} \quad \text { at } y=0, \\
& u=0 \quad \text { at } y=b, \\
& T=T_{w}(t) \quad \text { at } y=b .
\end{aligned}
$$

For convenience, we nondimensionalize (la) and (1b) as follows:

$$
\begin{aligned}
\frac{\partial \bar{u}}{\partial \tau} & =\frac{\partial^{2} \bar{u}}{\partial \xi^{2}}+\theta, \\
\operatorname{Pr} \cdot \frac{\partial \theta}{\partial \tau} & =\frac{\partial^{2} \theta}{\partial \xi^{2}}
\end{aligned}
$$

and the initial and boundary conditions may be stated as

$$
\begin{aligned}
& \bar{u}=0 \quad \text { at } \tau=0, \\
& \theta=0 \quad \text { at } \tau=0, \\
& \bar{u}=0 \quad \text { at } \xi=0, \\
& \theta=0 \quad \text { at } \xi=0, \\
& \bar{u}=0 \quad \text { at } \xi=1, \\
& \theta=\phi(\tau) \quad \text { at } \xi=1,
\end{aligned}
$$

where

$$
\begin{aligned}
\theta & =\frac{T-T_{\infty}}{T_{\infty}}, \\
\bar{u} & =\frac{v}{g b^{2} \beta T_{\infty}} u, \\
\xi & =\frac{y}{b}, \\
\tau & =\frac{v}{b^{2}} t, \\
\operatorname{Pr} & =\frac{v}{\alpha}, \\
\phi(\tau) & =\frac{T_{w}(\tau)}{T_{\infty}}-1 .
\end{aligned}
$$


By applying the integral transform [17] and convolution theorem to (5b), (6b), (7b), and (8b), we obtain the temperature solution $\theta(\xi, \tau)$ as

$$
\begin{aligned}
\theta(\xi, \tau)= & \frac{2 \pi}{\operatorname{Pr}} \sum_{m=1}^{\infty}(-1)^{m+1} m \sin (m \pi \xi) \\
& \cdot \int_{0}^{\tau} \phi(\tau-t) \exp \left(-\frac{m^{2} \pi^{2}}{\operatorname{Pr}} t\right) \mathrm{d} t .
\end{aligned}
$$

Since (10) includes the randomly fluctuating temperature $\phi(\tau)$, the temperature field must not be estimated deterministically but statistically. Therefore, the autocorrelation $R_{\theta}$ for $\theta$ is derived for the statistical estimation of the temperature field and can be expressed as follows:

$$
R_{\theta}\left(\xi_{1}, \xi_{2}, \tau, \tau+h\right)=\left\langle\theta\left(\xi_{1}, \tau\right) \cdot \theta\left(\xi_{2}, \tau+h\right)\right\rangle
$$

where $\langle\cdot\rangle,\left\{\xi_{1}, \xi_{2}\right\}$, and $h$ denote the expectation operator, a set of arbitrary coordinate values, and an arbitrary time interval, respectively. If $\phi(\tau)$ is a stationary random process, then $R_{\theta}$ is obtained by substituting (10) into (11) as follows:

$$
R_{\theta}\left(\xi_{1}, \xi_{2}, h\right)=\left(\frac{2 \pi}{\operatorname{Pr}}\right)^{2} \sum_{m=1}^{\infty} \sum_{n=1}^{\infty}(-1)^{m+n} m n \sin \left(m \pi \xi_{1}\right)
$$

$$
\begin{aligned}
R_{\bar{u}}\left(\xi_{1}, \xi_{2}, h\right)= & 4 \sum_{m=1}^{\infty} \sum_{n=1}^{\infty} \sin \left(m \pi \xi_{1}\right) \sin \left(n \pi \xi_{2}\right) \\
& \times \int_{0}^{1} \int_{0}^{1} \sin (m \pi p) \sin (n \pi q)\left[\int_{0}^{\infty} \int_{0}^{\infty} R_{\theta}(p, q, h+r-s) \exp \left(-m^{2} \pi^{2} r-n^{2} \pi^{2} s\right) \mathrm{d} r \mathrm{~d} s\right] \mathrm{d} p \mathrm{~d} q
\end{aligned}
$$

$$
\begin{aligned}
& \cdot \sin \left(n \pi \xi_{2}\right) \times \int_{0}^{\infty} \int_{0}^{\infty} R_{\phi}(h+r-s) \\
& \cdot \exp \left(-\frac{m^{2} \pi^{2}}{\operatorname{Pr}} r-\frac{n^{2} \pi^{2}}{\operatorname{Pr}} s\right) \mathrm{d} r \mathrm{~d} s,
\end{aligned}
$$

where $R_{\phi}$ denotes the autocorrelation of $\phi(\tau)$.

The natural convective flow due to the temperature field given by (10) must be estimated statistically. An analytical solution to the initial-boundary value problem for (5a), (6a), (7a), and (8a) has already been derived [18] as follows:

$$
\begin{gathered}
\bar{u}(\xi, \tau)=2 \sum_{m=1}^{\infty} \sin (m \pi \xi) \int_{0}^{1} \sin (m \pi x) \\
\cdot\left[\int_{0}^{\tau} \theta(x, \tau-t) \exp \left(-m^{2} \pi^{2} t\right) \mathrm{d} t\right] \mathrm{d} x .
\end{gathered}
$$

Since the flow velocity also fluctuates randomly because of the randomness of $\theta$, the autocorrelation of the vertical velocity $R_{\bar{u}}$ is derived as its statistic. As in the case of the autocorrelation for the temperature given by (11), $R_{\bar{u}}$ can be expressed as

$$
R_{\bar{u}}\left(\xi_{1}, \xi_{2}, \tau, \tau+h\right)=\left\langle\bar{u}\left(\xi_{1}, \tau\right) \cdot \bar{u}\left(\xi_{2}, \tau+h\right)\right\rangle .
$$

By substituting (13) into (14) and assuming the stationarity in time, we obtain where $R_{\theta}$ is given by (12).

The power spectrum densities for $\theta$ and $\bar{u}$ are obtained from the following Wiener-Khintchine equation [19]:

$$
\begin{aligned}
S_{i}(\xi, \omega)=\frac{1}{2 \pi} \int_{-\infty}^{\infty} R_{i}(\xi, \xi, h) \exp (-j \omega h) \mathrm{d} h, & \\
& i=\theta \text { or } \bar{u},
\end{aligned}
$$

where $j$ is an imaginary number and $\omega$ is the angular frequency.

\section{Numerical Results and Discussion}

Some special cases of (12) and (15) are of theoretical interest. We now examine the cases in which $\phi(\tau)$ is expressed as white noise or a stationary Markov process.

3.1. White Noise. White noise is the most basic model for stationary random processes and is often used for numerical computation because of its ease of mathematical treatment. $R_{\phi}$ is represented by Dirac's delta function [20] as

$$
R_{\phi}(h)=S_{0} \delta(h),
$$

where $S_{0}$ is a constant. In this case, the channel wall temperature includes all frequency components. In order to estimate statistically the temperature and flow velocity, we here focus attention on their mean square. The mean square is identical with the variance if $\langle\phi\rangle=0$. The mean square of temperature is obtained by setting $\xi_{1}=\xi_{2}=\xi$ and $h=0$ in (12) and then substituting (17) into it. For the mean square of flow velocity, setting $\xi_{1}=\xi_{2}=\xi$ and $h=0$ in (15) as well, we have

$$
\begin{aligned}
\left\langle\theta^{2}\right\rangle & =\frac{4 S_{0}}{\operatorname{Pr}} \sum_{m=1}^{\infty} \sum_{n=1}^{\infty} \frac{(-1)^{m+n} m n \sin (m \pi \xi) \sin (n \pi \xi)}{m^{2}+n^{2}} \\
\left\langle\bar{u}^{2}\right\rangle & =\frac{4 S_{0}}{\pi^{4}(\operatorname{Pr}+1)}
\end{aligned}
$$


TABLE 1: Relationship between the convergence of $\left\langle\theta^{2}\right\rangle / S_{0}$ and the number of terms, for $\operatorname{Pr}=0.7$.

\begin{tabular}{lccccc}
\hline$\xi$ & & Number of terms & $200 \times 200$ & 0.29117 \\
\\
\hline 0.2 & $20 \times 20$ & $50 \times 50$ & $100 \times 100$ & 1.2910 & $400 \times 400$ \\
0.4 & 0.29127 & 0.29118 & 0.29117 & 4.0267 & 4.29117 \\
0.6 & 1.2917 & 1.2911 & 1.2910 & 18.092 \\
0.8 & 4.0316 & 4.0275 & 4.0269 & 18.095 & 18.091 \\
\hline
\end{tabular}

$$
\begin{aligned}
& \cdot \sum_{m=1}^{\infty} \sum_{n=1}^{\infty} \frac{(-1)^{m+n} m \sin (m \pi \xi) \sin (n \pi \xi)}{n\left(m^{2}+n^{2}\right)^{2}} \\
& \cdot \frac{\left[\operatorname{Pr} \cdot m^{2}+(\operatorname{Pr}+2) n^{2}\right]}{\left(\operatorname{Pr} \cdot m^{2}+n^{2}\right)} .
\end{aligned}
$$

The infinite series in (18) are approximated with $\infty \rightarrow$ 200 to obtain four significant digits of the mean square of temperature and flow velocity (see Tables 1 and 2).

Figures 2 and 3 show the effects of the Prandtl number on the mean square values of the temperature and velocity, respectively. From Figure 2, it is evident that the temperature fluctuations are larger for a lower Prandtl number. This tendency can be easily explained as follows: if (5b) is considered to be the $1 \mathrm{D}$ heat conduction equation, then the Prandtl number is equivalent to the reciprocal of thermal diffusivity, and thus low Prandtl numbers promote the transmission of the randomness of the wall temperature into the fluid in the channel. The divergence of the mean square on the heated channel wall (i.e., $\xi=1$ ) is attributed to the white noise's feature of having an infinite variance. This divergence is also observed in the mean square temperature of solids when the boundary temperature is given by white noise $[18,21$, 22]. A sharp increase in the slope of the mean square near the channel wall results from high-frequency components [22].

Figure 3 shows that the mean square velocity has bellshaped profiles (convex upward), and it exhibits peak values in the vicinity of $\xi=0.6$. The details of the peak positions are discussed later. This figure indicates that the velocity fluctuations decrease with an increase in the Prandtl number; this tendency is consistent with Figure 2.

3.2. Stationary Markov Process. Stationary Markov processes have an exponential autocorrelation [23]. Thus, $R_{\phi}$ is expressed as

$$
R_{\phi}(h)=S_{0} \exp \left(-\frac{|h|}{\mu}\right),
$$

where $\mu$ is a positive constant and referred to as correlation time. In this case, the mean square of $\theta$ and $\bar{u}$ is given by

$$
\begin{aligned}
& \left\langle\theta^{2}\right\rangle=4 S_{0} \mu \sum_{m=1}^{\infty} \sum_{n=1}^{\infty} \frac{(-1)^{m+n} m n \sin (m \pi \xi) \sin (n \pi \xi)}{\operatorname{Pr}-\mu n^{2} \pi^{2}}\left[\frac{2 \operatorname{Pr}}{\left(m^{2}+n^{2}\right)\left(\operatorname{Pr}+\mu n^{2} \pi^{2}\right)}-\frac{\mu \pi^{2}}{\operatorname{Pr}+\mu m^{2} \pi^{2}}\right], \\
& \left\langle\bar{u}^{2}\right\rangle=4 S_{0} \mu \sum_{m=1}^{\infty} \sum_{n=1}^{\infty} \frac{(-1)^{m+n} m n \sin (m \pi \xi) \sin (n \pi \xi)}{\left(m^{2}+n^{2}\right)\left(\operatorname{Pr}-\mu n^{2} \pi^{2}\right)} F(m, n),
\end{aligned}
$$

$$
\begin{aligned}
& \text { where } \\
& \qquad \begin{aligned}
F(m, n) \\
=\frac{2 r^{2}\left[\operatorname{Pr} \cdot m^{2}+(\operatorname{Pr}+2) n^{2}\right]}{(\operatorname{Pr}+1) n^{2}\left(m^{2}+n^{2}\right) \pi^{4}\left(\operatorname{Pr}+\mu n^{2} \pi^{2}\right)\left(\operatorname{Pr} \cdot m^{2}+n^{2}\right)} \\
\quad-\frac{\mu^{2}\left[\mu \pi^{2}\left(m^{2}+n^{2}\right)+2\right]}{\left(\operatorname{Pr}+\mu m^{2} \pi^{2}\right)\left(1+\mu n^{2} \pi^{2}\right)\left(1+\mu m^{2} \pi^{2}\right)} .
\end{aligned}
\end{aligned}
$$

Tables 3 and 4 show the convergence performance of the solutions. One can see that the Markov process makes the convergence of solutions worse than white noise. This tendency is particularly visible in the mean square of temperature. Even for $\infty \rightarrow 3200$, four significant digits are not obtained. In contrast, an approximation of $\infty \rightarrow 50$ is sufficient to obtain four significant digits in the mean square velocity. Therefore, considering the balance between computation accuracy and computation time, the infinite series in (20) and (21) are approximated with $\infty \rightarrow 3200$, leading to three significant digits of the mean square temperature.

Figures 4 and 5 show the mean square distributions of the temperature and flow velocity for different Prandtl numbers and correlation time, respectively. Because multiplying the Prandtl number by $k$ is equivalent to multiplying the correlation time by $1 / k$ in $\left\langle\theta^{2}\right\rangle$ (see (20)), identical curves are obtained for different combinations of $\operatorname{Pr}$ and $\mu$ in Figure 4. This fact means that the spatial distribution of $\left\langle\theta^{2}\right\rangle / S_{0}$ depends only on a new parameter $\operatorname{Pr} / \mu$. A comparison among the results for a fixed value of $\mu$ indicates that a decrease in the Prandtl number increases the temperature fluctuation; this is the same as in the case of white noise. On the other hand, a comparison among the results for a fixed value of $\mathrm{Pr}$ 
TABLE 2: Relationship between the convergence of $\left\langle\bar{u}^{2}\right\rangle / S_{0}$ and the number of terms, for $\operatorname{Pr}=0.7$.

\begin{tabular}{cccccc}
\hline$\xi$ & & \multicolumn{3}{c}{ Number of terms } & $200 \times 200$ \\
\hline 0.2 & $20 \times 20$ & $50 \times 50$ & $100 \times 100$ & $2.7880 \cdot 10^{-3}$ & $2.7880 \cdot 10^{-3}$ \\
0.4 & $2.7879 \cdot 10^{-3}$ & $2.7880 \cdot 10^{-3}$ & $2.7880 \cdot 10^{-3}$ & $8.7664 \cdot 10^{-3}$ & $8.7664 \cdot 10^{-3}$ \\
0.6 & $8.7657 \cdot 10^{-3}$ & $8.7663 \cdot 10^{-3}$ & $8.7664 \cdot 10^{-3}$ & $1.2337 \cdot 10^{-2}$ & $1.2337 \cdot 10^{-2}$ \\
0.8 & $1.2335 \cdot 10^{-2}$ & $1.2337 \cdot 10^{-2}$ & $1.2337 \cdot 10^{-2}$ & $8.4651 \cdot 10^{-3}$ & $8.4651 \cdot 10^{-3}$ \\
\hline
\end{tabular}

TABLE 3: Relationship between the convergence of $\left\langle\theta^{2}\right\rangle / S_{0}$ and the number of terms, for $\operatorname{Pr}=0.7$ and $\mu=1$.

\begin{tabular}{lccccc}
\hline$\xi$ & & Number of terms & $3200 \times 3200$ & $6400 \times 6400$ \\
\hline 0.2 & $400 \times 400$ & $300 \times 800$ & $1600 \times 1600$ & $3.7173 \cdot 10^{-2}$ & 0.14889 \\
0.4 & $0.7092 \cdot 10^{-2}$ & $0.148710^{-2}$ & $3.7161 \cdot 10^{-2}$ & 0.14892 \\
0.6 & 0.33596 & 0.33657 & 0.14884 & 0.33703 & 0.33711 \\
0.8 & 0.60639 & 0.60826 & 0.60920 & 0.60967 & 0.60991 \\
\hline
\end{tabular}

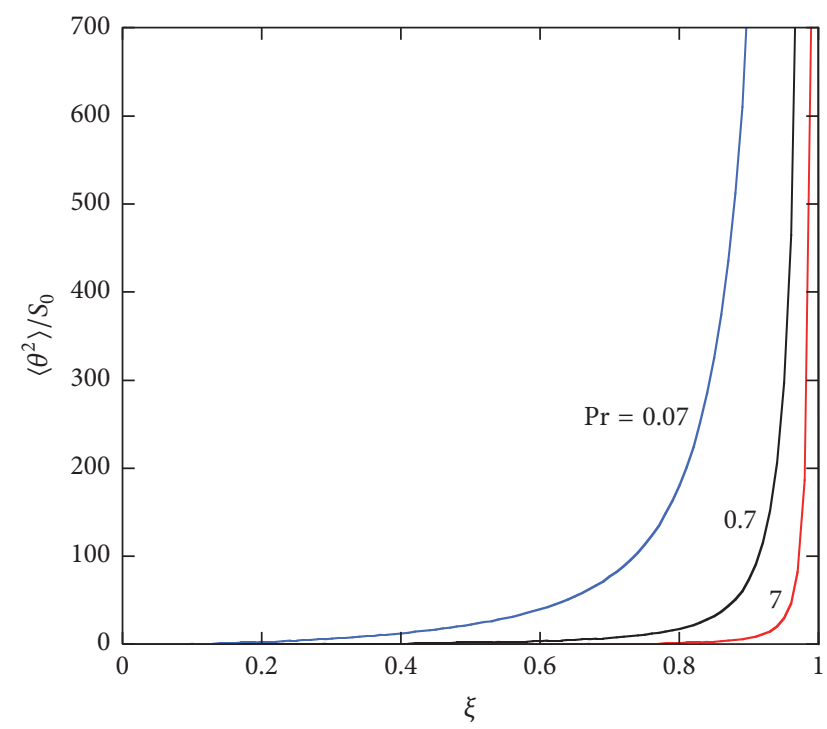

FIGURE 2: Mean square distribution of temperature for $\phi(\tau)$ of white noise.

shows that a short correlation time reduces the temperature fluctuation of the fluid. This is because a short correlation time increases the fraction of high-frequency components in the wall temperature fluctuation, which decay rapidly with increasing distance from the wall.

Figure 5 demonstrates that the distribution tendency of $\left\langle\bar{u}^{2}\right\rangle / S_{0}$ is essentially the same as in Figure 3. Interestingly, a clear difference in the mean square velocity is observed between the parameter sets for which the identical mean square distribution of temperature is obtained, for example, $(\operatorname{Pr}, \mu)=(0.7,1)$ and $(0.07,0.1)$. In comparison between those parameter sets, the mean square velocity is smaller for a shorter correlation time.

The peak positions of the mean square distributions of velocity are tabulated in Table 5 for different Prandtl numbers. In the case of the Markov process, a decrease in the

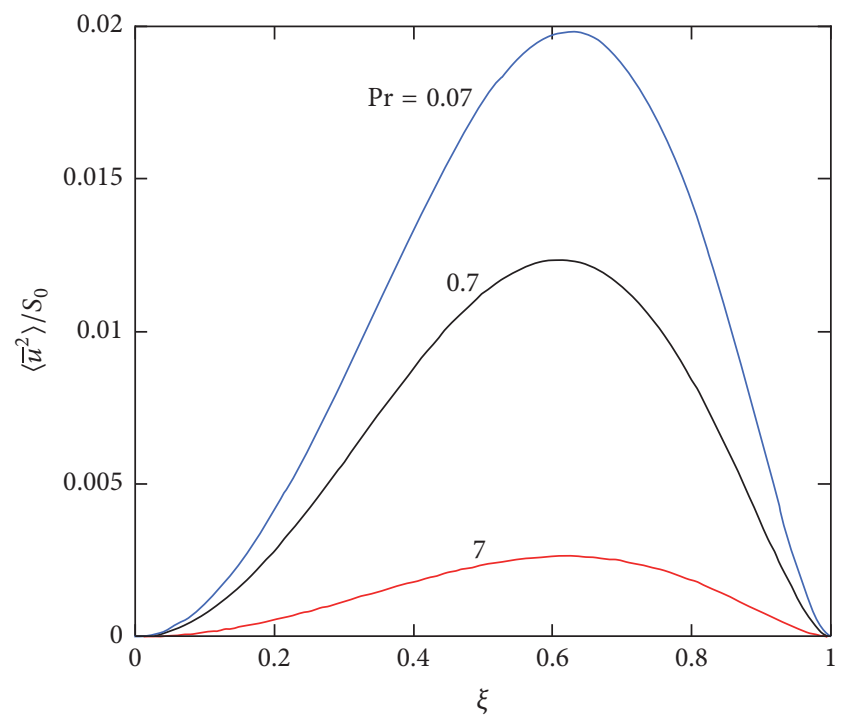

FIgURE 3: Mean square distribution of vertical velocity for $\phi(\tau)$ of white noise.

correlation time slightly increases the $\xi$-coordinate values of the peak position for all the Prandtl numbers. In addition, because white noise has zero correlation time [24], it yields larger $\xi$-coordinate values than the Markov process. However, roughly speaking, $\xi \approx 0.6$ in any of these cases.

\section{Concluding Remarks}

A stochastic natural convection analysis was presented based on the correlation theory approach. The laminar natural convection of viscous fluid was studied in an asymmetrically heated vertical channel that is composed of doubly infinite plates. Analytical solutions of the autocorrelations for the temperature and flow velocity were derived under the condition that the random fluctuation in the channel wall temperature can be modelled as a stationary random 
TABLE 4: Relationship between the convergence of $\left\langle\bar{u}^{2}\right\rangle / S_{0}$ and the number of terms, for $\operatorname{Pr}=0.7$ and $\mu=1$.

\begin{tabular}{cccccc}
\hline$\xi$ & & \multicolumn{3}{c}{ Number of terms } & $200 \times 200$ \\
\hline 0.2 & $20 \times 20$ & $50 \times 50$ & $100 \times 100$ & $9.0025 \cdot 10^{-4}$ & $9.0025 \cdot 10^{-4}$ \\
0.4 & $2.0018 \cdot 10^{-4}$ & $9.0025 \cdot 10^{-4}$ & $9.0025 \cdot 10^{-4}$ & $2.7633 \cdot 10^{-3}$ & $2.7633 \cdot 10^{-3}$ \\
0.6 & $2.7630 \cdot 10^{-3}$ & $2.7633 \cdot 10^{-3}$ & $2.7633 \cdot 10^{-3}$ & $3.6248 \cdot 10^{-3}$ & $3.6248 \cdot 10^{-3}$ \\
0.8 & $3.6241 \cdot 10^{-3}$ & $3.6247 \cdot 10^{-3}$ & $3.6248 \cdot 10^{-3}$ & $2.0536 \cdot 10^{-3}$ & $2.0536 \cdot 10^{-3}$ \\
\hline
\end{tabular}

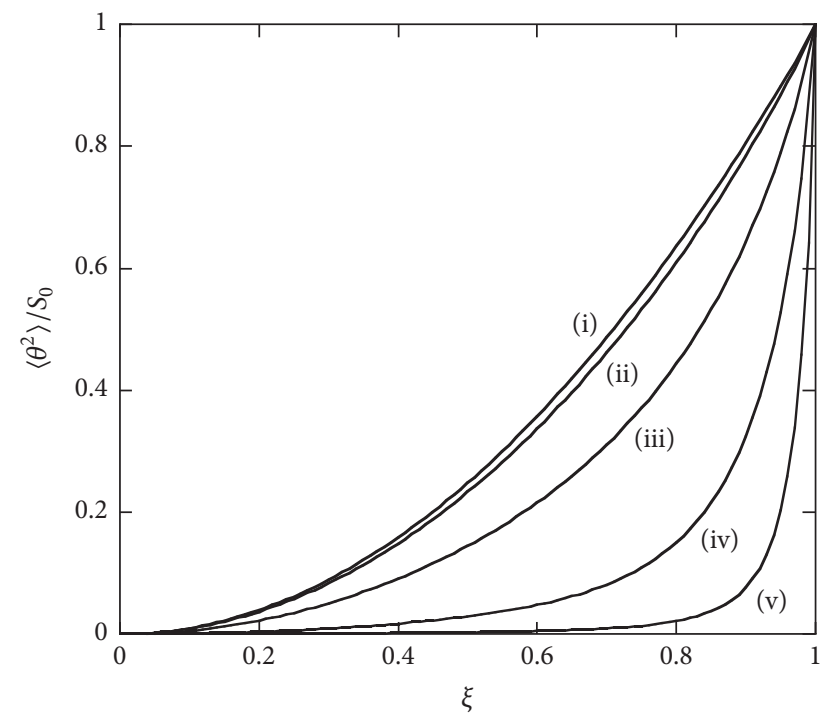
$(\operatorname{Pr}, \mu)$
(i) $(0.07,1)$
(ii) $(0.7,1),(0.07,0.1)$
(iii) $(7,1),(0.7,0.1),(0.07,0.01)$
(iv) $(7,0.1),(0.7,0.01)$
(v) $(7,0.01)$

FIGURE 4: Mean square distribution of temperature for $\phi(\tau)$ of a stationary Markov process.

TABLE 5: $\xi$ coordinate values at which the maximum mean square velocity is observed.

\begin{tabular}{|c|c|c|c|c|c|}
\hline \multirow{2}{*}{$\operatorname{Pr}$} & \multicolumn{4}{|c|}{$\mu$ (stationary Markov process) } & \multirow{2}{*}{ White noise } \\
\hline & 0.01 & 0.1 & 1 & $\infty$ & \\
\hline 0.07 & 0.6147 & 0.5875 & 0.5786 & 0.5773 & 0.6247 \\
\hline 0.7 & 0.6069 & 0.5883 & 0.5784 & 0.5773 & 0.6114 \\
\hline 7 & 0.6205 & 0.6093 & 0.5853 & 0.5773 & 0.6212 \\
\hline
\end{tabular}

process. Numerical calculations were performed for the wall temperature given as white noise or a stationary Markov process. Numerical results demonstrated the effects of the Prandtl number and the correlation time of the random process on the mean square values of the temperature and flow velocity. The main conclusions are summarised as follows:

(i) A decrease in the Prandtl number or an increase in the correlation time of the random process increases the mean square velocity but does not change its spatial distribution tendency, which is a bell-shaped profile

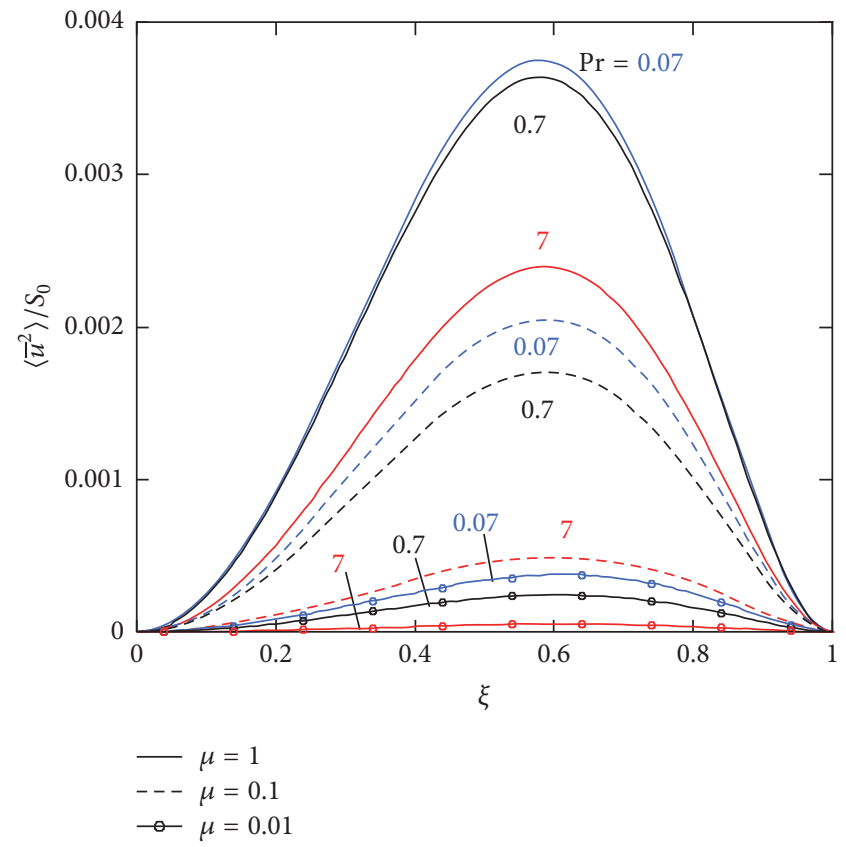

Figure 5: Mean square distribution of vertical velocity for $\phi(\tau)$ of a stationary Markov process.

with a peak at a certain horizontal distance from the channel wall.

(ii) The peak position is not substantially affected by the Prandtl number or the correlation time. When measured with the dimensionless coordinate, $\xi$, the position is $\xi \approx 0.6$, and no more than about $10 \%$ of shift in position occurs depending on the values of those parameters.

\section{Nomenclature}

$b: \quad$ Distance between two plates, $\mathrm{m}$

g: Acceleration due to gravity, $\mathrm{m} / \mathrm{s}^{2}$

$h$ : Dimensionless time interval

$j$ : $\quad$ Imaginary number

$k$ : $\quad$ Arbitrary positive real number

Pr: $\quad$ Prandtl number

$R_{\phi}, R_{\theta}, R_{\bar{u}}:$ Autocorrelations

$S_{\theta}, S_{\bar{u}}: \quad$ Power spectrum densities

$S_{0}: \quad$ Positive constant

$t: \quad$ Time, $s$

T: $\quad$ Local temperature, $\mathrm{K}$ 
$u$ : Vertical velocity, $\mathrm{m} / \mathrm{s}$

$\bar{u}$ : Dimensionless vertical velocity

$x$ : Vertical coordinate, $\mathrm{m}$

$y$ : Horizontal coordinate, $\mathrm{m}$.

\section{Greek Symbols}

$\alpha$ : Thermal diffusivity, $\mathrm{m}^{2} / \mathrm{s}$

$\beta$ : Thermal expansion coefficient, $1 / \mathrm{K}$

$\delta$ : Dirac's delta function

$\phi$ : Random function with respect to time

$\mu$ : Dimensionless correlation time

$v$ : Kinematic viscosity, $\mathrm{m}^{2} / \mathrm{s}$

$\theta$ : Dimensionless local temperature

$\tau$ : Dimensionless time

$\omega$ : Dimensionless angular frequency

$\xi$ : Dimensionless horizontal coordinate.

\section{Subscripts}

$w$ : Value on the wall at $y=b$

$\infty$ : Value in the ambient fluid.

\section{Conflicts of Interest}

The author declares that there are no conflicts of interest regarding the publication of this article.

\section{References}

[1] H. Miyata, S. Iijima, R. Ooshima, T. Abe, T. Hisamatsu, and T. Hamamatsu, "Application technology on ceramics for structural components of high temperature machines," Transactions of the Japan Society of Mechanical Engineers Series A, vol. 54, no. 505, pp. 1700-1708, 1988.

[2] D. E. Hutchinson and M. P. Norton, "Applicability of stochastic process theory to heat conduction in solids with random temperature fields," Applied Energy, vol. 22, no. 4, pp. 241-269, 1986.

[3] R. A. Heller and S. Thangjitham, "Probabilistic methods in thermal stress analysis," in Thermal Stresses, Elsevier Science, R. B. Hetnarski, Ed., pp. 190-268, New York, NY. USA, 1987.

[4] R. Chiba, "Stochastic analysis of heat conduction and thermal stresses in solids: a review," in Heat Transfer Phenomena and Applications, InTech, S. N. Kazi, Ed., Rijeka, Croatia, 2012.

[5] A. Campo and T. Yoshimura, "Random heat transfer in flat channels with timewise variation of ambient temperature," International Journal of Heat and Mass Transfer, vol. 22, no. 1, pp. 5-12, 1979.

[6] A. G. Madera and A. N. Sotnikov, "Method for analyzing stochastic heat transfer in a fluid flow," Applied Mathematical Modelling, vol. 20, no. 8, pp. 588-592, 1996.

[7] M. Kamiński, "Stochastic problem of viscous incompressible fluid flow with heat transfer," ZAMM, vol. 81, pp. 827-837, 2001.

[8] O. P. Le Maitre, M. T. Reagan, H. . Najm, R. G. Ghanem, and O. M. Knio, "A stochastic projection method for fluid flow: II. Random process," Journal of Computational Physics, vol. 181, no. 1, pp. 9-44, 2002.

[9] B. Ganapathysubramanian and N. Zabaras, "Sparse grid collocation schemes for stochastic natural convection problems,"
Journal of Computational Physics, vol. 225, no. 1, pp. 652-685, 2007.

[10] B. Schieche and J. Lang, "Uncertainty quantification for thermoconvective Poiseuille flow using stochastic collocation," International Journal of Computational Science and Engineering, vol. 9, no. 5-6, pp. 465-477, 2014.

[11] T. P. Sommer, R. M. C. So, and H. S. Zhang, "Heat transfer modeling and the assumption of zero wall temperature fluctuations," Transactions - ASME: Journal of Heat Transfer, vol. 116, no. 4, pp. 855-863, 1994.

[12] E. Semma, V. Timchenko, M. El Ganaoui, and E. Leonardi, "The effect of wall temperature fluctuations on the heat transfer and fluid flow occuring in a liquid enclosure," International Journal of Heat and Fluid Flow, vol. 26, no. 4, pp. 547-557, 2005.

[13] B. M. Nicolai and J. De Baerdemaeker, "Simulation of heat transfer in foods with stochastic initial and boundary conditions," Trans IChemE, Food and Bioproducts Processing, vol. 70 (Part C), pp. 78-82, 1992.

[14] Y. Joshi and B. Gebhart, "Vertical transient natural convection flows in cold water," International Journal of Heat and Mass Transfer, vol. 27, no. 9, pp. 1573-1582, 1984.

[15] T. Paul, B. K. Jha, and A. K. Singh, "Transient free convective flow in a vertical channel with constant temperature and constant heat flux on walls," Heat and Mass Transfer/Waermeund Stoffuebertragung, vol. 32, no. 1-2, pp. 61-63, 1996.

[16] A. K. Singh, H. R. Gholami, and V. M. Soundalgekar, "Transient free convection flow between two vertical parallel plates," Heat and Mass Transfer/Waerme- und Stoffuebertragung, vol. 31, no. 5, pp. 329-331, 1996.

[17] M. N. Ozisik, Boundary value problems of heat conduction, New York, NY, USA, Dover, 1989.

[18] J. C. Samuels, "Heat conduction in solids with random external temperatures and/or random internal heat generation," International Journal of Heat and Mass Transfer, vol. 9, no. 4, pp. 301314, 1966.

[19] D. R. Axelrad, Stochastic Mechanics of Discrete Media, Springer Berlin Heidelberg, Berlin, Heidelberg, 1993.

[20] V. Krishnan, Probability and random processes, John Wiley and Sons, Hoboken, 2nd edition, 2015.

[21] S. Amada, "Thermal stresses in bodies with random temperature distribution at their boundaries," Zairyo/Journal of the Society of Materials Science, vol. 31, no. 342, pp. 251-257, 1982.

[22] R. Chiba and Y. Sugano, "Stochastic thermoelastic problem of a functionally graded plate under random temperature load," Archive of Applied Mechanics, vol. 77, no. 4, pp. 215-227, 2007.

[23] R. F. Stengel, Optimal Control and Estimation, Dover, New York, NY, USA, 1994.

[24] G. A. Pavliotis, Stochastic processes and applications: diffusion processes, the Fokker-Planck and Langevin equations, Texts in Applied Mathematics, Springer, New York, NY, USA, 2014. 


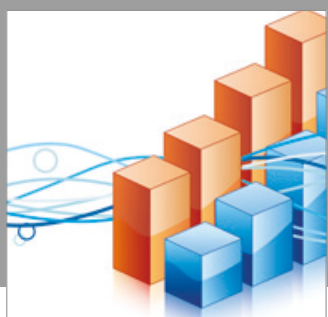

Advances in

Operations Research

vatersals

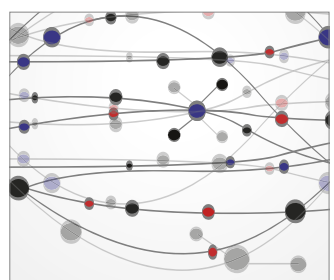

\section{The Scientific} World Journal
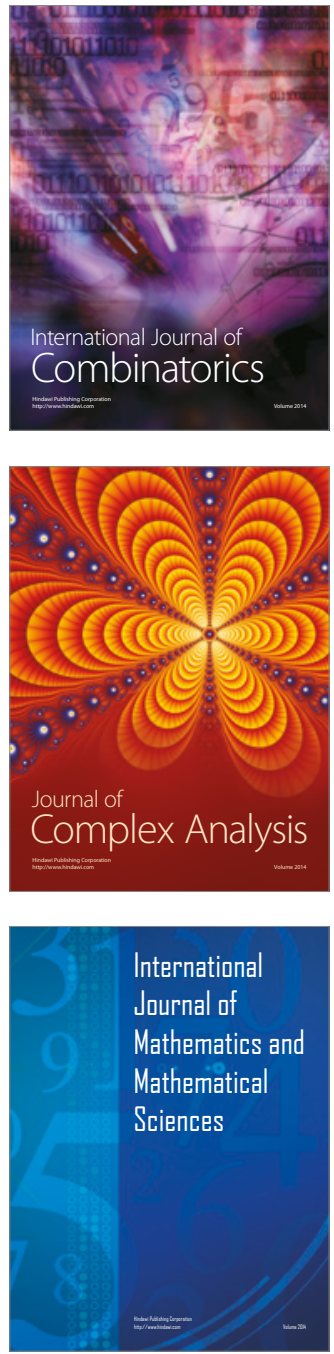
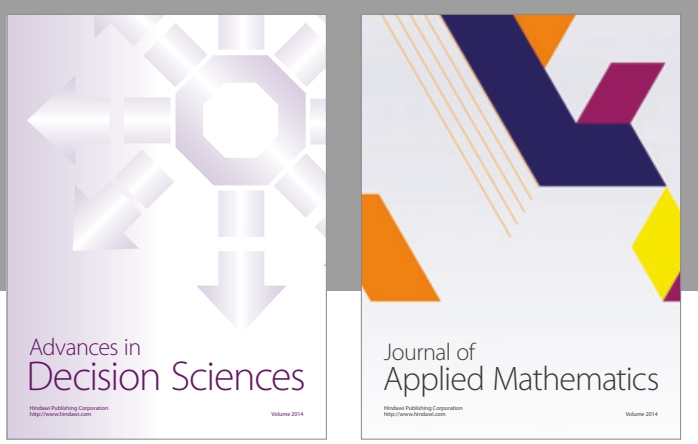

Algebra

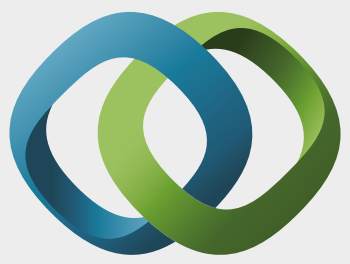

\section{Hindawi}

Submit your manuscripts at

https://www.hindawi.com
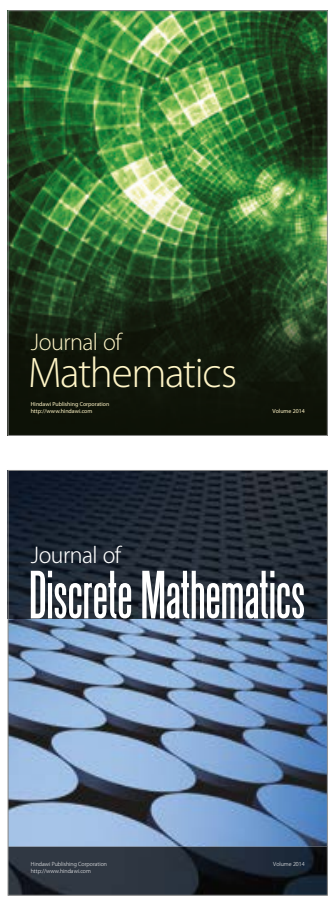

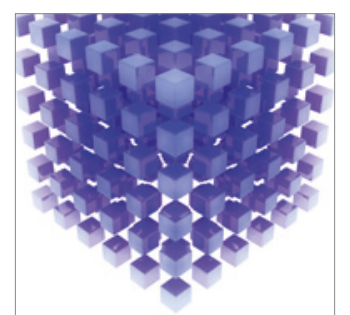

Mathematical Problems in Engineering
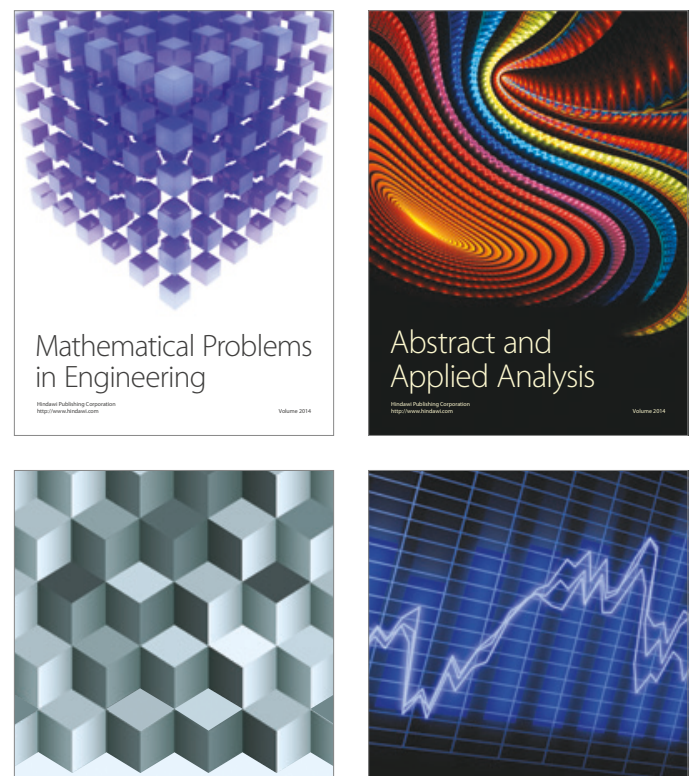

Journal of

Function Spaces

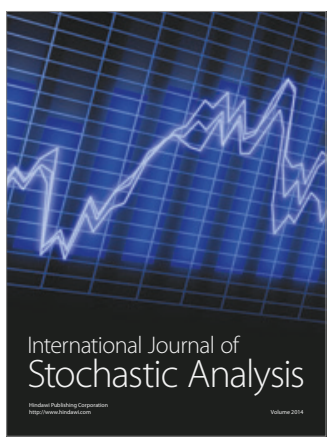

Probability and Statistics
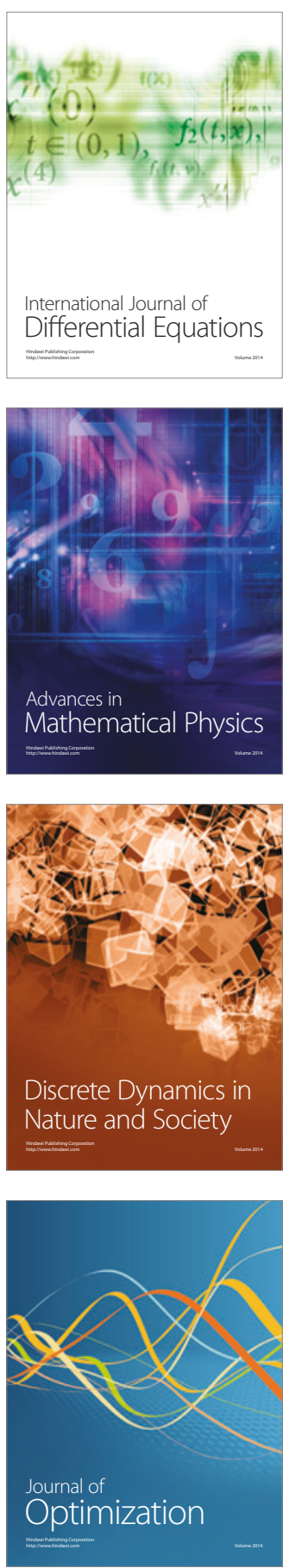